\title{
AN APPLICATION OF GEOMETRICAL ISOMETRIES IN NON-PLANAR POLYCYCLIC MOLECULES (CORANNULENE, SUMANENE, FULLERENE)
}

\author{
Miroslava Ivanova ${ }^{1} \&$ Lilko Dospatliev $^{2}$ \\ ${ }^{1}$ Department of Informatics and Mathematics, Faculty of Economics, Trakia University \\ Student campus, Stara Zagora 6000, Bulgaria, e-mail: mivanova@uni-sz.bg \\ ${ }^{2}$ Department of Pharmacology, Animal Physiology and Physiological Chemistry, Faculty of \\ Veterinary Medicine, Trakia University \\ Student campus, Stara Zagora 6000, Bulgaria, e-mail: Ikd@abv.bg
}

\begin{abstract}
Geometry is widely used in chemistry. In this paper we introduce a novel methodology to transmit the origin to the center of a polygon in a polycyclic molecule (corannulene, sumanene, or fullerene) such that the special axis be perpendicular to the plane containing the polygon. The mathematical calculations are described completely and the algorithm will be showed as a computer program.
\end{abstract}

Keywords: Frame, Isometry, Orthogonal transformation, Polygon, Non-planar polycyclic molecule.

\section{INTRODUCTION}

An isometry is a distance-preserving injective map between metric spaces. The isometries associated with the Euclidean metric, are called Euclidean motions, which forms a Lie group under composition. This group is among the oldest and most studied implicitly, long before the concept of group was invented.

One of the applications of isometries is to transfer or rotate the coordinate system in order to simplify the computations or visions. This usually happens in all branches of sciences which apply the analytic geometry.

In computational chemistry, sometimes, one needs to calculate some properties in each point on the area of molecule or above and below it, so one must put ghost $(\mathrm{Bq})$ atom in an arbitrary point, exactly. Evaluation of the aromaticity, antiaromaticity and nonaromaticity of compounds by nucleus independent chemical shift criterion (NICS), is an example for it. To NICS calculation at each point above and below of the all polygons, one must put some $\mathrm{Bq}$ atoms in various distances on the $z$ axis, straightforwardly [3]. In non-planar molecule, vertically putting $\mathrm{Bq}$ atoms in various distances of the rings in different sheets is not very hard, but estimation of components of the nuclear magnetic shielding tensors is very hard and for more complex molecules is impossible.

Using proposed method and doing calculation separately for each polygon facilitate estimation of nuclear magnetic shielding tensors components $[3,1,10]$. Based to this technique in [7] the authors investigated the evaluation of aromaticity of some non-planar molecules.

In this work we study of the geometric structure of some molecules such as Corannulene and Sumanene which are polycyclic aromatic hydrocarbons and molecules which have polygons in their structure for example Fullerenes. Our goal is by mathematical language we transfer the origin to the center of a pentagon (or hexagon) in the space, such that the z-axes to be perpendicular to the plane containing the polygon (or hexagon).

\section{IRTIIE Vol. 6, No. 1, 2018 ISSN 1314-8788 (print), ISSN 1314-8796 (online), doi: 10.15547/artte.2018.01.004}




\section{IRTILC}

Ipplied Resseirlches in Teednicis, Technologies and Eductation

Journal of the Faculty of Technics and Technologies, Trakia University https://sites.google.com/a/trakia-uni.bg/artte/

\section{METHODS}

In this section, we shall investigate the isometries of Euclidean space, and see how two frames uniquely determine an isometry.

Definition 2.1 ([6]). An isometry, or rigid motion, of Euclidean space is a mapping that preserves the Euclidean distance $d(\mathbf{p}, \mathbf{q})=\|\mathbf{p}-\mathbf{q}\|$ between points $\mathbf{p}, \mathbf{q} \in \mathbf{R}^{3}$. More precisely, an isometry of $\mathbf{R}^{3}$ is a mapping $F: \mathbf{R}^{3} \rightarrow \mathbf{R}^{3}$ such that

$$
d(F(\mathbf{p}), F(\mathbf{q}))=d(\mathbf{p}, \mathbf{q})
$$

for all point $\mathbf{p}, \mathbf{q}$ in $\mathbf{R}^{3}$.

Definition 2.2 ([6]). A set $\mathbf{e}_{1}, \mathbf{e}_{2}, \mathbf{e}_{3}$ of three mutually orthogonal unit vectors tangent to $\mathbf{R}^{3}$ at $\mathbf{p}$ is called a frame at the point $\mathbf{p}$.

Thus $\mathbf{e}_{1}, \mathbf{e}_{2}, \mathbf{e}_{3}$ is a frame if and only if

$$
\begin{aligned}
& \mathbf{e}_{1} \cdot \mathbf{e}_{1}=\mathbf{e}_{2} \cdot \mathbf{e}_{2}=\mathbf{e}_{3} \cdot \mathbf{e}_{3}=1, \\
& \mathbf{e}_{1} \cdot \mathbf{e}_{2}=\mathbf{e}_{1} \cdot \mathbf{e}_{3}=\mathbf{e}_{2} \cdot \mathbf{e}_{3}=0 .
\end{aligned}
$$

For example, $\mathbf{i}=(1,0,0), \mathbf{j}=(0,1,0), \mathbf{k}=(0,0,1)$ is a frame at each point of $\mathbf{R}^{3}$ which is called the standard frame. It is clear that at each point of the Euclidean space, there exist uncountable frames. Depending on the application, certain frames are used. For example in local curve theory, the Frenet frame [5], determines the geometric properties of the curve. Here we use the frames to obtain an important isometry. First we state a vital theorem in differential geometry, see [6] for example.

Theorem 2.3 ([6]). Given any two frames on $\mathbf{R}^{3}$, say $\mathbf{e}_{1}, \mathbf{e}_{2}, \mathbf{e}_{3}$ at the point $\mathbf{p}$ and $\mathbf{f}_{1}, \mathbf{f}_{2}, \mathbf{f}_{3}$ at the point $\mathbf{q}$, there exists a unique isometry $F$ of $\mathbf{R}^{3}$ such that $F *\left(\mathbf{e}_{i}\right)=\mathbf{f}_{i}$ for $1 \leq i \leq 3$.

To compute the isometry $F$ in the above theorem, let $e_{i}=\left(a_{i 1}, a_{i 2}, a_{i 3}\right), f_{i}=\left(b_{i 1}, b_{i 2}, b_{i 3}\right)$, $A=\left(a_{i j}\right), B=\left(b_{i j}\right)$, where $A$ and $B$ are the attitude matrices of the frames $\left\{e_{i}\right\}$ and $\left\{f_{i}\right\}$, respectively. According to [6] $C=B^{t} A$ is an orthogonal transformation and $C\left(e_{i}\right)=f_{i}$. If $T$ be the translation by the point $q-C(p)$, then $F=T \circ C$ is the desired isometry.

\section{EXPERIMENTAL}

Corannulene is a polycyclic aromatic hydrocarbon with one central pentagonal ring and five peripheral hexagonal rings (Figure 1). Sumanene is a polycyclic aromatic hydrocarbon with one central hexagonal ring and three peripheral hexagonal and three peripheral pentagonal rings, alternately (Figure 2). Fullerenes are a family of carbon allotropes which composed entirely of carbon, in the form of a sphere, ellipsoid, cylinder, or tube. The structure of fullerenes is composed of hexagonal, pentagonal or sometimes heptagonal and octagonal rings $[2,8,9]$ (Figure 3 ). 


\section{IRTITE

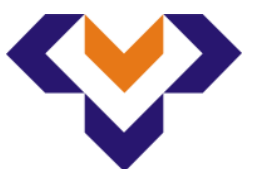 \\ Ipplied Resseirlohes in Technics, Technologies and Bductation \\ Journal of the Faculty of Technics and Technologies, Trakia University https:///ites.google.com/a/trakia-uni.bg/artte/}

We apply the Theorem 2.3 to transfer the origin and the standard frame to the center of an arbitrary pentagon or hexagon in a polycyclic molecule (corannulene, sumanene, or fullerene), such that the z-axis will be perpendicular on this polygon. To do so, we need a frame on the center point of polygon.



Figure 1. Structure of Corannulene

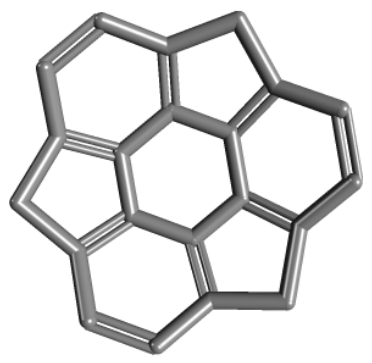

Figure 2. Structure of Sumanene

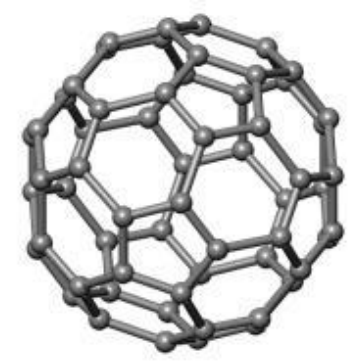

Figure 3. Structure of Fullerene

We describe the method for a hexagon, the case pentagon is similar.

Let $u_{1}, u_{2}$ and $u_{3}$ are three consecutive vertices of the hexagon. Then the vector $\overrightarrow{u_{1} u_{2}} \times \overrightarrow{u_{1} u_{3}}$ is perpendicular to the plane containing the hexagon. Dividing this vector by its own length, we have the unit vector

$$
v_{3}=\frac{\overrightarrow{u_{1} u_{2}} \times \overrightarrow{u_{1} u_{3}}}{\left|\overrightarrow{u_{1} u_{2}} \times \overrightarrow{u_{1} u_{3}}\right|}
$$

To get the unit vector $v_{2}=v_{3} \times v_{1}$ we multiply $v_{3}$ by the unit vector $v_{1}=\frac{\overrightarrow{u_{1} u_{2}}}{\left|\overrightarrow{u_{1} u_{2}}\right|}$. Then the resulting set $\left\{v_{1}, v_{2}, v_{3}\right\}$ is a frame.

To obtain an isometry $F$ which maps the frame $\left\{v_{1}, v_{2}, v_{3}\right\}$ to the standard frame $\{i, j, k\}$, let the vectors $v_{1}, v_{2}, v_{3}$ have the following coordinates $v_{1}=\left(a_{11}, a_{12}, a_{13}\right), v_{2}=\left(a_{21}, a_{22}, a_{23}\right)$, $v_{3}=\left(a_{31}, a_{32}, a_{33}\right)$, the matrix of their coordinates is denoted by $A=\left(a_{i j}\right)$ and let $B$ is the identity matrix, so

$$
C=B^{t} A=A=\left(\begin{array}{lll}
a_{11} & a_{12} & a_{13} \\
a_{21} & a_{22} & a_{23} \\
a_{31} & a_{32} & a_{33}
\end{array}\right)
$$

In other words, if $p=(x, y, z)^{t}$ be the primary coordinate of the point $p$, then its new coordinate $(X, Y, Z)^{t}$ is given by

$$
F(p)=\left(\begin{array}{l}
X \\
Y \\
Z
\end{array}\right)=\left(\begin{array}{lll}
a_{11} & a_{12} & a_{13} \\
a_{21} & a_{22} & a_{23} \\
a_{31} & a_{32} & a_{33}
\end{array}\right)\left(\begin{array}{l}
x \\
y \\
z
\end{array}\right) .
$$

IRTIIE Vol. 6, No. 1, 2018 ISSN 1314-8788 (print), ISSN 1314-8796 (online), doi: 10.15547/artte.2018.01.004 


\section{ARTITE Ipplied Resseirl ches in Teechnics, Technologies and Eductition Journal of the Faculty of Technics and Technologies, Trakia University https://sites.google.com/a/trakia-uni.bg/artte/}

\section{RESULTS}

All calculations of coordinate transformation we performed in MATLAB environment. This program code has been shown in the following lines

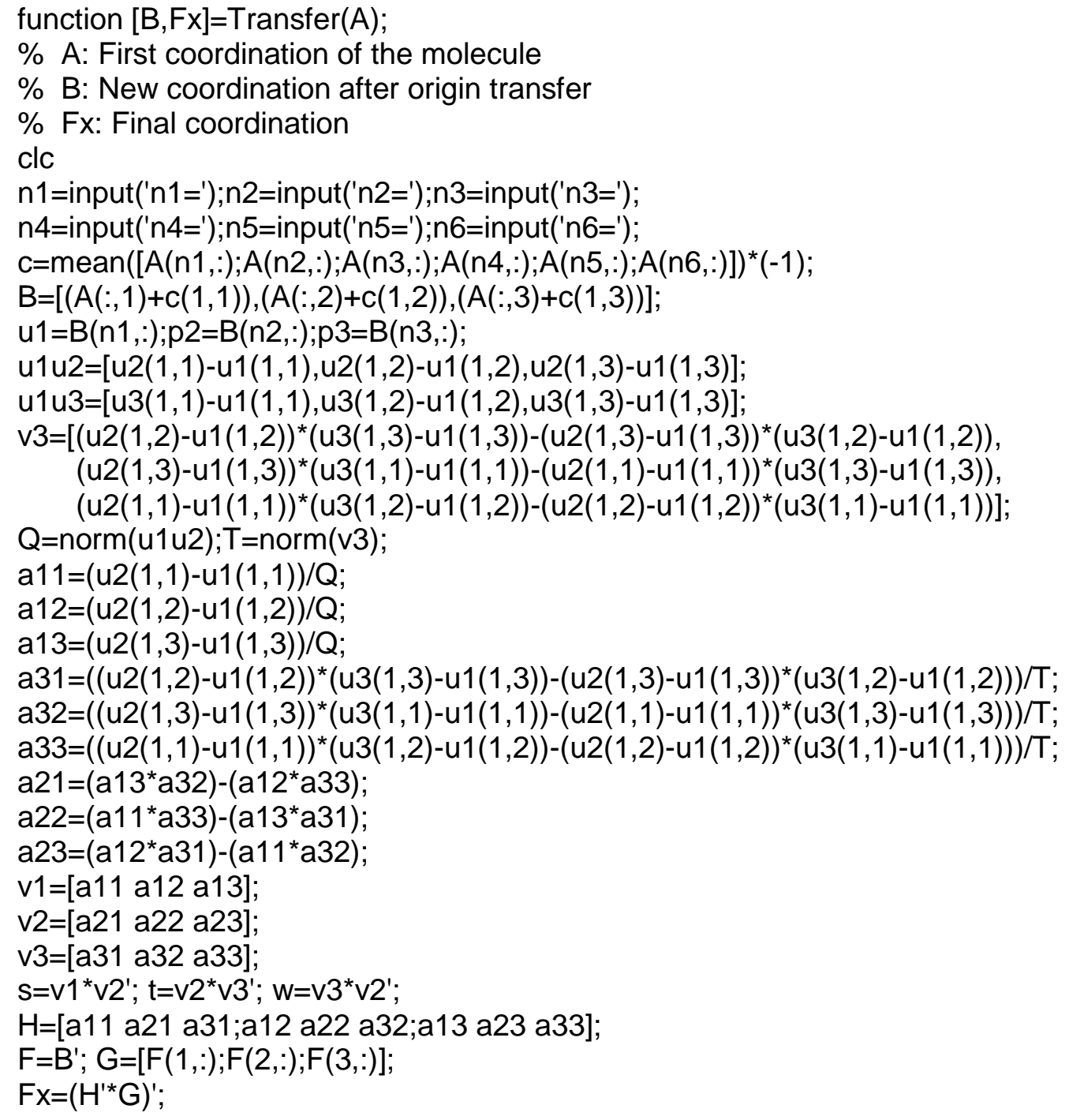

As an example we apply the program code for Corannulene molecule. Figure 4 shows the structure of molecule before and after translating and rotating the coordinate system. 

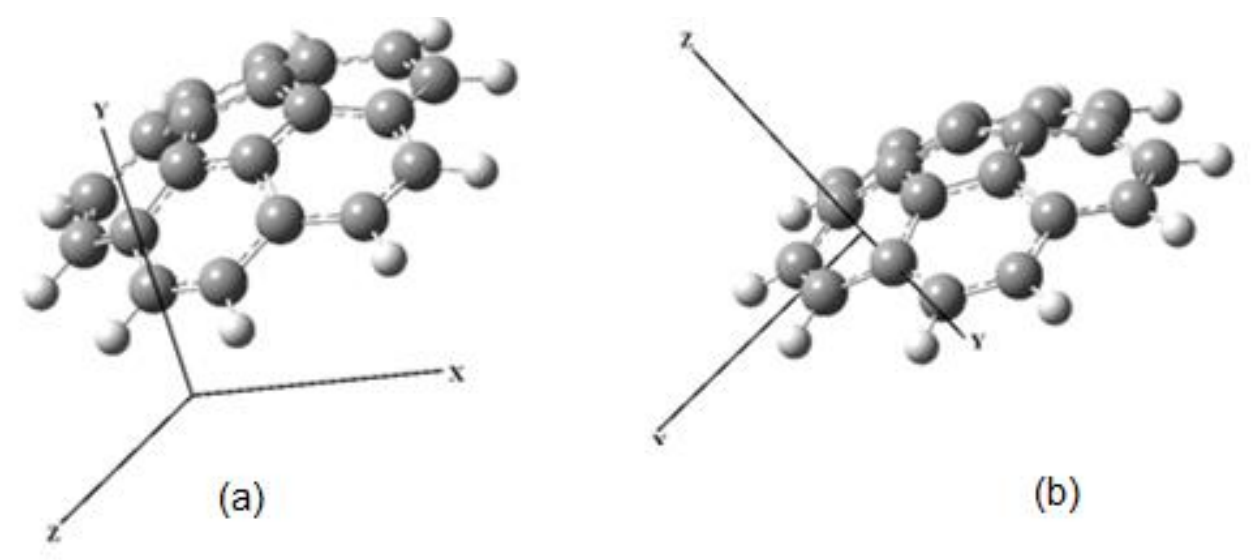

(b)

Figure 4. Corannulene molecule: (a) origin coordinate in in arbitrary point, (b) molecule was rotated with uncertain angle such that $z$-axis is perpendicular to hexagon

\section{CONCLUSIONS}

Proposed methodology in this work helps ones to transmit origin of coordinate to an arbitrary point and changes the axes coordinate direction perpendicular to an arbitrary polygon. It facilitates estimation of components of the nuclear magnetic shielding tensors in non-planar molecules and can be used for any calculation that needs to such coordinate change. Although our discussion was based on z-axis, but it can be used for other axes by a simple rotation.

\section{REFERENCES}

[1] Barth W.E. \& Lawton R.G. (1966). Dibenzo[ghi,mno]fluoranthene, J. Am. Chem. Soc., Vol. 88, No. 2, (1966), pp. 380-381.

[2] Cataldo F., Graovac A. \& Ori O. (2011). The Mathematics and Topology of Fullerenes, Springer, Verlag, Berlin, (2011).

[3] Chen Z., Wannere C.S., Corminboeuf C., Puchta R. \& Schleyer P.V.R. (2005). Nucleusindependent chemical shifts (NICS) as an aromaticity criterion, Chemical Reviews - Columbus, Vol. 105, No. 10, (2005), pp. 3842-3888.

[4] Fallah-Bagher-Shaidaei H., Wannere C.S., Corminboeuf C., Puchta R. \& Schleyer P.V.R. (2006). Which NICS aromaticity index for planar rings is best?, Organic Letters, Vol. 8, No. 5, (2006), pp. 863-866.

[5] Millman R. \& Parker G. (1997). Elements of Differential Geometry, Prentice-Hall, (1977).

[6] O'Neill B. (1997). Elementary Differential Geometry, 2nd ed., Academic Press, Elsevier, (1997).

[7] Reisi-Vanani A. \& Rezaei A. A. (2015). Evaluation of the aromaticity of non-planar and bowl-shaped molecules by NICS criterion, J. Mol. Graph. Model., Vol. 61, (2015), pp. 85-88.

[8] Rezaei A.A. (2014). Polygonal tiling of some surfaces containing fullerene molecules, Iranian J. Math. Chem., Vol. 5, No. 2, (2014), pp. 99-105.

[9] Rezaei A.A. (2015). Tiling fullerene surface with heptagon and octagon, Fullerenes, Nanotubes and Carbon Nanostructures, Vol. 23, No. 12, (2015), pp. 1033-1036.

[10] Sakurai H., Daiko T. \& Hirao T. (2003). A synthesis of sumanene, a fullerene fragment, Science, Vol. 301, No. 5641, (2003), pp. 1878-1878. 